Revista

Actualidades Investigativas

en Educación

\title{
Modelo de transferencia de conocimiento a través de la gamificación: Un gcMooc
}

Model of knowledge transfer through gamification: a gcMooc

Volumen 19, Número 2

Mayo-Agosto

pp. 1-25

\section{Antonia Terán Bustamante Bertha Mendieta Jiménez}

\section{Cite este documento así}

Terán Bustamante, Antonia. y Mendieta Jiménez, Bertha. (2019). Transferencia de conocimiento a través de la gamificación: Un gcMooc. Revista Actualidades Investigativas en Educación, 19(2), 1-25. Doi. 10.15517/aie.v19i2.36997 


\title{
Modelo de transferencia de conocimiento a través de la gamificación: Un gcMooc Model of knowledge transfer through gamification: a gcMooc
}

\author{
Antonia Terán Bustamante ${ }^{1}$ \\ Bertha Mendieta Jiménez ${ }^{2}$
}

\begin{abstract}
Resumen: Actualmente los Massive Open Online Course (MOOC) son una pieza esencial en la educación a distancia. El objetivo del presente artículo es analizar un modelo de transferencia de conocimiento a través de un gcMOOC de emprendimiento, desde su desarrollo hasta la terminación por parte del alumnado. La creación del conocimiento se basa en estrategias creativas de aprendizaje, tales como: la gamificación, las diversas interacciones entre los participantes a través de redes, foros y comunidades de práctica. La estrategia metodológica utilizada en esta investigación es mixta, cuantitativa y cualitativa, con alcance descriptivo. La población objeto de estudio son 1910 personas, de las cuales 890 (46.60\%) participaron al inicio y finalizaron 216 (24.3\%), se les aplicaron dos cuestionarios. La estructura del curso que se analiza está basada en el conectivismo, en la cooperación y en la gamificación. Los resultados muestran un mayor porcentaje de retención del alumnado mediante la introducción de un diseño de escenario educativo basado en juegos mediante contenidos altamente visuales y retos intelectuales. Las evidencias muestran que en la realización de un curso de este tipo se deben trabajar mecanismos de entrega y calidad del contenido. Sin embargo, hay que considerar cursos de tiempos cortos. Se concluye que la realización de un MOOC no es tarea sencilla, sino que involucra muchas herramientas pedagógicas para lograr una mayor retención.
\end{abstract}

Palabras clave: conocimiento, educación, gamificación, innovación, mooc

Abstract: In recent years, the MOOC (Massive Open Online Course) is an essential piece of distance education. The aim of this research article to analyze a knowledge transfer model through an entrepreneurial MOOC, where the basis of the course for knowledge creation is creative strategies of learning as gamification, communities of practice. The methodological approach used in this research is mixed, quantitative and qualitative with scope descriptive. The population under study is 1910 people, of which 890 (46.60\%) participated at the beginning and 216 (24.3\%) finalized, to which it uses two questionnaires. The course structure is connectivism, cooperation, and gamification, with a practical methodology of game-based learning to develop the spirit of enterprise. The results show a higher percentage of student retention through the introduction of an educational scenario design based on games, through visual contents and intellectual challenges. At the same time, the evidence shows that in the realization of this course it is necessary to work on mechanisms of delivery and quality of material must be worked on and presented in short times. The research concluded that the understanding of a MOOC is not an easy task; it involves many pedagogical tools to achieve higher retention.

Keywords: educational, knowledge, innovation, gamification, mooc

\footnotetext{
1 Profesora-investigadora de la Universidad Panamericana, Escuela de Ciencias Económicas y Empresariales, México. Dirección electrónica: ateran@up.edu.mx

2 Profesora de la Universidad Panamericana, Facultad de Ingeniería, México. Dirección electrónica: bmendieta@up.edu.mx
}

Artículo recibido: 16 de octubre, 2018

Enviado a corrección: 29 de enero, 2019

Aprobado: $1^{\circ}$ de abril, 2019 


\section{Introducción}

En un mundo tan dinámico como el actual, la adquisición de conocimiento y su gestión son cada día más relevantes para las organizaciones y para las personas. De acuerdo con lo anterior, la innovación educativa, se hace presente en los procesos y en en el desarrollo de la tecnología (Ramírez Montoya, 2008). Dentro de las principales innovaciones educativas, García-Peñalvo, Fidalgo-Blanco y Sein-Echaluce (2015) proponen un mapa de tendencias organizado en cuatro zonas conjuntas: 1. Perspectiva institucional, 2. Perspectiva del profesorado; 3. Desarrollo de competencias trasversales, y 4. Perspectiva de extensión institucional, donde se sitúan los MOOC en la intersección de estas cuatro áreas. Actualmente los MOOC's están detonando una nueva cultura del aprendizaje masivo, el cual incluye la personalización colectiva, aprendizaje individual, y heterogeneidad como fuente potencial para generar nuevos conocimientos en redes (Uden, Sinclair, Tao y Liberona, 2014).

De acuerdo con Rivera (2014) y Renninger, Cai, Lewis, Adams y Ernst (2011), el éxito en la transferencia de conocimiento en un ambiente virtual depende de las fortalezas y necesidades de las personas participantes como de la relación de estos y el contenido del curso. Aunado a lo anterior, hay que considerar que el aprendizaje es un proceso de conexiones, de nodos y fuentes de información especializada que pueden residir en múltiples dispositivos y actores (Ramírez Montoya, 2008). Aunado a lo anterior, las tecnologías de la información y comunicación (TIC) están transformando la educación, configurando nuevos modos de trabajo, de búsqueda de información y de aprendizaje colaborativo (García Retana, 2011). La manera en la que el conocimiento se transfiere de las instituciones de educación superior a la sociedad varía según el tipo de conocimiento que es transferido y de los mecanismos que se han establecido para tal fin.

En la práctica, la enseñanza y la investigación son las formas básicas a través de las cuales el conocimiento se crea y se transfiere (Bayona y González, 2010). Los entornos donde se da esa enseñanza-aprendizaje pueden ser presenciales o virtuales, en el que cada modalidad tendrá su propia complejidad de interacción. Hoy, estas innovaciones están transformando las instituciones educativas, tanto en la forma en la que se aprende como a nivel estructural (Torres-Díaz, Infante y Valdiviezo, 2014). Una de las transformaciones que está experimentando la educación, apoyada por el desarrollo tecnológico, es la impulsada por las universidades más prestigiosas del mundo, y se encamina a configurar lo que Vest 
(2006) denomina, a nivel sistémico: la meta-universidad, con alcance global y sustentada por cuatro elementos: i) las conexiones entre universidades, ii) las conexiones entre participantes, iii) los contenidos y iv) el acceso abierto.

En la actualidad las conexiones entre universidades están soportadas a través de las redes de computadores a escala mundial, existen muchas redes de universidades que comparten cursos, profesorado e inclusive estudiantes (Torres-Díaz et al., 2014). Las conexiones entre participantes son un aspecto muy relevante, así las poblaciones estudiantiles crean sus propias redes para interactuar y socializar, al utilizar wikis, blogs, redes sociales y sistemas de mensajería entre otros (Cabrero, Llorente y Vázquez, 2014). En lo que respecta a los contenidos, estos abarcan una amplia gama de temas y el acceso es abierto a toda persona que lo quiera tomar. Sin embargo, hay que considerar que el MOOC demanda la adquisición de conocimientos y habilidades, donde el modelo constructivista de aprendizaje se centra en la población estudiantil, quienes son responsables de conectar y construir el conocimiento en un contexto de redes y grupos (Hernández-Carranza, RomeroCorella y Ramírez-Montoya, 2015).

Los MOOC's son un nuevo enfoque formativo en la educación superior, fundamentada en la apertura, la masividad, la ubicuidad y por el momento, en la gratuidad. En su esencia, suponen una evolución del aprendizaje abierto (Open Learning Movement), que se caracteriza por un modelo de enseñanza global y participativa, dirigido a una cantidad ilimitada de participantes y del establecimiento de más conexiones y más posibilidades de aprendizaje para un curso determinado. La presente investigación tiene como objetivo estudiar y analizar un curso de emprendimiento creativo en línea (e-learning) a nivel internacional a través de una plataforma MOOC: Thinking Outside The Box: Creative Entrepreneurship. Este tipo de curso en una plataforma MOOC genera un gran valor para la sociedad, las organizaciones y las personas.

La promoción del emprendimiento contribuye al desarrollo social, crecimiento económico, mejora el espíritu empresarial y a la vez puede ayudar a la sociedad a superar tiempos de crisis. Gracias a la apertura de este tipo de cursos masivos abiertos en línea, una gran cantidad de participantes puede obtener una formación empresarial. El presente trabajo se desarrolla a través de tres secciones: en la primera se exponen las bases conceptuales sobre la transferencia del conocimiento, los cursos masivos en línea (MOOC) y la relación que tienen con la gamificación. En la segunda sección se presenta la estrategia 
metodológica empleada en la investigación y se detalla la población objeto de estudio. Finalmente, en la tercera sección se muestran los resultados obtenidos, a través de una descripción mixta cuantitativa y cualitativa (estadística descriptiva) de cómo se diseñó y gestionó este curso de emprendimiento y cuáles fueron los resultados de todas las interacciones con los actores involucrados.

\section{Referente Teórico}

Los MOOC han generado un gran interés como agentes de cambio en la educación superior. De acuerdo con Zheng y Yang (2017), la investigación enfocada en los MOOC presenta un rápido crecimiento concentrandose principalmente en el campo de la pedagogía e informática, sin embargo, hay pocos casos empíricos y el contenido carece de la contribución a la construcción de un sistema teórico. Para Porter (2015), el futuro de los MOOC es mucho menos predecible de lo que se podría suponer, en virtud de que nadie puede decir con precisión si cumplirán el cambio prometido al sistema de educación superior o si se trata de una burbuja educativa que estallará en cualquier momento. Con los MOOC, la educación superior se ha convertido por primera vez en un producto de internet y está abierta a las mismas presiones que afectan otras industrias como la de la música y las publicaciones, y con resultados muy impredecibles.

Lo que es muy notable acerca de esta tendencia en innovación tecnológica y educativa es la velocidad con la que el público en general ha aceptado los MOOC como parte del panorama educativo. Muchas universidades apenas han tenido tiempo de entender los principales sistemas MOOC y sus primeros modelos de negocios, y de comenzar a planificar su propia respuesta, antes de que surjan otros nuevos proveedores. Uno de los mayores desafíos para todos los involucrados en los MOOC, incluidas las grandes plataformas, es avanzar a un ritmo adecuado de cambio: seguir moviéndose lo suficientemente rápido para mantener el impulso, pero no tan rápido como para que muchos posibles participantes se queden atrás (Pomerol, Epelboin y Thoury, 2015).

\subsection{Transferencia del conocimiento a través del e-learning}

De acuerdo con García Retana (2011) y Ruíz (2016) hay una creciente tendencia a considerar que la educación a distancia será, en un futuro inmediato, un elemento fundamental de los sistemas educativos, así como una estrategia que puede ofrecer una solución de calidad a los problemas de acceso e igualdad de educación para todos. Los 
sistemas educativos han sido impactados fuertemente por las tecnologías de información y comunicación (TIC). La educación a distancia se ha hecho sustentable gracias a las TIC, ha conformado nuevas formas de aprender y al mismo tiempo ha establecido nuevas prácticas de enseñanza. Durante años, las instituciones de educación superior han utilizado internet y otras tecnologías digitales para el desarrollo y transferencia de educación (Organización para la Cooperación y el Desarrollo Económicos [OCDE], 2008). Al modificar y transformar las prácticas tradicionales se ha requerido involucrar a diversos actores con el objetivo de generar aprendizajes en espacios y tiempos novedosos.

De acuerdo con lo anterior, la formación a distancia tiene y tendrá un gran impacto social, al ofrecer una amplia gama de posibilidades, a su vez permite otorgar independencia, flexibilidad y libertad al aprendizaje, además de poder desempeñar un papel excepcional en la ampliación del alcance de los sistemas educativos (Yee, 2012). El término de transferencia del conocimiento describe las actividades destinadas a trasladar un conocer -un conocimiento- (Terán-Bustamante y Chavarría Olarte, 2014) un saber qué, para qué y cómo, es decir es compartir saberes, que se conviertan en competencias. Es una forma de crear valor. Para Holi, Wickramasinghe y Leeuwen (2008) la transferencia de conocimiento es el proceso mediante el cual el conocimiento, la experiencia y los activos intelectuales de las universidades son aplicados constructivamente más allá de sus límites para el beneficio de la economía y la sociedad.

La transferencia de conocimiento a través de ambientes virtuales es un proceso más elaborado que el presencial, requiere de la participación de más actores y de más recursos creativos, lo cual es posible gracias a la existencia de una serie de estructuras y mecanismos que crean, contienen y trasladan el conocimiento. Al integrar tecnologías digitales en la formación superior, el MOOC conlleva la necesidad de hacer explícita una transformación del papel del docente universitario de transmisor, hacia la facilitador y evaluador de nuevas experiencias de formación masiva en línea. Resulta importante considerar que, en esa transferencia de conocimiento, no solo hay que saber qué es un $\mathrm{MOOC}$, hay que saber qué no es, cómo se aprende, su estrategia, la metodología y cuáles son los medios para llevar a cabo esa transferencia de conocimiento. 


\subsection{Los cursos masivos en línea (MOOC) y su tipología}

Los cursos en línea masivos y abiertos (Massive Online Open Course, MOOC) son un nuevo enfoque formativo en la educación superior. Diseñados en torno a un objetivo de aprendizaje de un área de conocimiento específico (Educase, 2011). Los MOOC existen desde hace algunos años como una herramienta de aprendizaje colaborativo (Pernías y Lujan, 2014). El término MOOC fue acuñado por Dave Cormier y Bryan Alexander (Siemens, 2012). Aunque existe una gran cantidad de literatura, las características y definición de un MOOC se encuentran aún en discusión. Para efectos de esta investigación, se toma la conceptualización de McAuley, Stewart, Siemens y Cormier (2010, p.5) quienes menciona que

un MOOC integra la conectividad de las redes sociales, la facilitación de un reconocido experto en un campo de estudio y una colección de recursos en línea de libre acceso. Se basa en la participación activa de cientos o miles de estudiantes que auto-organizan su participación de acuerdo con los objetivos de aprendizaje, el conocimiento previo habilidades e intereses comunes.

Existen diferentes tipos de MOOC's, de acuerdo con los objetivos, metodologías y resultados que se esperan. Hay diversas formas de clasificarlos, entre las más conocida se tiene la de Siemens (2012) y Daniel (2012) que distinguen tres tipos de MOOC: I) los conectivistas cMOOC, II) los de contenido xMOOC y III) los quasiMOOC. En los conectivistas cMOOC, la riqueza está en el número de relaciones que se establecen y en el contenido que viaja entre cada nodo. Las relaciones constituyen la base del trabajo colaborativo en la población estudiantil crea y desarrolla una tarea conjunta, lo que conlleva a que construyan significados (Álvarez y Bassa, 2013; Torres-Díaz et al., 2014) (ver Tabla 1).

Los tipos de contenido xMOOC y los quasiMOOC toman los cursos antiguos del elearning, sin embargo, Clark (2013) elaboró una nueva taxonomía -muy específica- dividida en ocho categorías que pretende diferenciar más ampliamente y anticipar el tipo de experiencias de formación masiva a distancia que pueden ir surgiendo en el futuro. Por su parte, Vázquez, López y Sarasola (2013) incorporan el tMOOC, que es una combinación xMOOC y el cMOOC, pero poniendo énfasis en las tareas (ver Tabla 1). El gcMOOC se enfoca en la gamificación, tiene cuatro factores clave: i) factor tecnológico, II) estrategia formativa, III) factores cooperativos que representan la parte más conectivista del curso, y IV) 
la gamificación la cual interactúa con el resto de factores y su finalidad es fomentar la motivación (Oriol-Borras, 2015) (ver Tabla 1).

Tabla 1.

Tipos de MOOC

\begin{tabular}{ll}
\hline Autor & Tipo de MOOC \\
\hline Siemens (2012) & 1. cMOOC \\
Daniel (2012) & (conectivismo)
\end{tabular}

(conductismo/ clásico)

El elemento más relevante son las tareas a las que la población estudiantil debe dar respuesta, y ser capaz de movilizar diferentes tipos de competencias vinculadas a la resolución de problemas de diversa índole. Aprendizaje activo y participativo a partir de compartir el conocimiento con los demás (colaboración orientada a redes). Implica relación con el resto de la comunidad del curso. Resalta el aprender haciendo.

Aprendizaje lineal, individualizado y guiado, centrado en la información que transmite el docente. Estos cursos se caracterizan porque uno de sus elementos curriculares más relevantes son los contenidos, que son evaluados a través de pruebas de evaluación de corrección automática. Conservar la figura del equipo docente como gestor del conocimiento y de los contenidos prácticos y teóricos que utilizará el alumnado. Esto supone un cambio sustancial con el modelo anterior, donde se ponía el énfasis en la colaboración de todos los participantes como parte fundamental del método de enseñanza.

3. quasiMOOC Toma los cursos de e-learning y los sube a una plataforma MOOC.

\begin{tabular}{|c|c|c|}
\hline \multirow[t]{5}{*}{ Clark (2013) } & 1. transferMOOC & $\begin{array}{l}\text { Consisten en tomar los cursos existentes en las } \\
\text { Universidades de e-learning y transferirlos a una } \\
\text { plataforma MOOC. } \\
\text { Experiencias desarrolladas previamente a través de } \\
\text { LMS que se reformulan para desarrollarse a través de } \\
\text { un formato MOOC. }\end{array}$ \\
\hline & 2. madeMOOC & $\begin{array}{l}\text { Incorporan elementos de vídeo, hacen hincapié en la } \\
\text { calidad de la creación de tareas que debe realizar el } \\
\text { alumnado, potencian el trabajo entre iguales y la } \\
\text { coevaluación. Intensa utilización de los documentos } \\
\text { audiovisuales y Multimedia y altamente interactivos e } \\
\text { innovadores. }\end{array}$ \\
\hline & 3. synchMOOC & $\begin{array}{l}\text { Los cursos estructurados que presentan fechas } \\
\text { específicas de comienzo y de finalización, así como de } \\
\text { realización de las evaluaciones. }\end{array}$ \\
\hline & 4. asynchMOOC & Altamente flexibles y sin fecha de inicio ni finalización. \\
\hline & 5. adaptiveMOOC & $\begin{array}{l}\text { Utilizan algoritmos adaptativos para presentar } \\
\text { experiencias de aprendizaje personalizadas, basadas } \\
\text { en la evaluación dinámica y la recopilación de datos del } \\
\text { curso. Se analizan grandes cantidades de datos para } \\
\text { personalizar, recogidos a través de diferentes } \\
\text { estrategias de evaluación. }\end{array}$ \\
\hline
\end{tabular}




\begin{tabular}{|c|c|c|}
\hline Autor & Tipo de MOOC & Concepto \\
\hline & 6. groupMOOC & $\begin{array}{l}\text { Elaborados para grupos específicos. Experiencias } \\
\text { focalizadas en el desarrollo de actividades realizadas en } \\
\text { grupos de trabajo. }\end{array}$ \\
\hline & 7. connectivistMOOC & $\begin{array}{l}\text { Procesos educativos en los que se promueve el } \\
\text { desarrollo de conexiones entre las personas } \\
\text { participantes. }\end{array}$ \\
\hline & 8. miniMOOC/ SPOC & $\begin{array}{l}\text { Experiencias de formación breves sobre contenidos } \\
\text { concretos. }\end{array}$ \\
\hline $\begin{array}{l}\text { Vázquez-Cano y } \\
\text { López-Meneses } \\
\text { (2014); Scopeo } \\
\text { (2013) }\end{array}$ & $\begin{array}{l}\text { 1. tMOOC (Mixto: } \\
\text { Conectivismo y } \\
\text { conductismo) }\end{array}$ & $\begin{array}{l}\text { Centrado en las tareas, modelo híbrido que adopta } \\
\text { planteamientos del } x M O O C \text { y el } c M O O C \text {, hace énfasis } \\
\text { en la resolución de determinados tareas y actividades } \\
\text { para poder ir avanzando a lo largo del curso. }\end{array}$ \\
\hline $\begin{array}{l}\text { Fidalgo Blanco, } \\
\text { Sein-Echaluce, } \\
\text { Borrás Gené y } \\
\text { Oriol y García } \\
\text { Peñalvo, (2014) }\end{array}$ & $\begin{array}{l}\text { 1. Modelo cooperativo } \\
\text { xcMOOC }\end{array}$ & $\begin{array}{l}\text { Modelo cooperativo recoge características de ambos } \\
\text { dos tipos de } \mathrm{MOOC} \text {, los } \mathrm{x} \text { y los } \mathrm{c} \text {. }\end{array}$ \\
\hline $\begin{array}{l}\text { Fidalgo Blanco, } \\
\text { Sein-Echaluce, } \\
\text { Borrás Gené y } \\
\text { Oriol y García } \\
\text { Peñalvo, (2014); } \\
\text { García-Peñalvo, } \\
\text { Fidalgo-Blanco } \\
\text { y Sein-Echaluce } \\
\text { (2015 y 2017) }\end{array}$ & $\begin{array}{l}\text { 1. gcMOOC (su } \\
\text { enfoque es la } \\
\text { gamificación). }\end{array}$ & $\begin{array}{l}\text { Este MOOC parte del modelo cooperativo de Fidalgo et } \\
\text { al. (2014), posee características de ambos tipos de } \\
\text { MOOC, los x y los c. La estructura de este tiene } 4 \\
\text { factores clave: i) Factor tecnológico, II) Estrategia } \\
\text { formativa, III) Factores cooperativos, que representan la } \\
\text { parte más conectivista del curso, y se da a través de la } \\
\text { interacción y cooperación de todos los actores } \\
\text { incluyendo los participantes y IV) la gamificación. }\end{array}$ \\
\hline Porter (2015) & $\begin{array}{l}\text { 1. COOC (Curso } \\
\text { Corporativo } \\
\text { Abierto en Línea,) } \\
\text { 2. SPOC (Pequeño } \\
\text { curso privado } \\
\text { abierto o en } \\
\text { línea.) }\end{array}$ & $\begin{array}{l}\text { Este MOOC al contrario del MOOC y COOC, el objetivo } \\
\text { de un SPOC es ofrecer a un pequeño grupo de } \\
\text { personas un curso a la medida. }\end{array}$ \\
\hline
\end{tabular}

Fuente: Elaboración propia con base en Oriol-Borras, Núñez y Blanco, (2014); Cabrero et al., (2014); Fidalgo et al., 2014; Daniel (2012); Clark (2013); Siemens (2015); SCOPEO (2013); Pernias y Lujan (2014); Vázquez-Cano y López Meneses (2014) Porter (2015) y García-Peñalvo, Fidalgo-Blanco y Sein-Echaluce (2015 y 2017).

\subsection{Los MOOC y la gamificación}

Los MOOC's ofrecen nuevas oportunidades de enseñanza, pero tienen sus limitaciones, como son las elevadas tasas de ausentismo y/o abandono, por lo que se ha recurrido a crear espacios donde se favorezcan las interacciones entre la población participante como son: las comunidades virtuales de práctica, los foros on line, el uso de redes sociales y la realización de actividades que fomenten el involucramiento, el reto, el juego y la diversión como la gamificación, en un enfoque más conectivista. Para poder lograr atraer a más usuarios los MOOC están recurriendo a la gamificación, la cual tiene que ver 
con la aplicación de conceptos que se encuentran habitualmente en el juego y en actividades lúdicas como la de los videojuegos.

Zichermann y Cunningham (2011, p.11) conceptualizan la gamificación como "un proceso relacionado con el pensamiento del jugador y las técnicas de juego para atraer a los usuarios y resolver problemas". De acuerdo con Kapp (2012, p.15), la gamificación se puede conceptualizar como "la aplicación de mecánicas, dinámicas y estéticas de juegos en entornos no lúdicos", con el fin de que las personas adquieran conocimientos y adopten comportamientos, es decir una experiencia significativa a través de la acción, para promover el aprendizaje y resolver problemas. Para Werbach y Hunter (2015) la gamificación tiene tres niveles según lo táctico o conceptual que sea el elemento; estos son: dinámicas, mecánicas y componentes que, junto a la estética del juego, crearán la experiencia. Las dinámicas son el concepto, la estructura implícita que tiene que ver con las motivaciones intrínsecas que impulsan a seguir jugando. Las mecánicas son los procesos que empujan el desarrollo y son el elemento clave principal generador de lealtad -retos, competencias, cooperación, recompensas, retroalimentación-y los componentes son las implementaciones específicas de las dinámicas y mecánicas -niveles, puntos, logros-.

Oriol-Borras (2015) afirma que la gamificación proporciona experiencias que permiten activar la motivación por el aprendizaje, tienen retroalimentación constante que permite un aprendizaje más significativo y una mayor retención en la memoria al ser más atractivos y generar competitividad a la vez que colaboración. De acuerdo con lo anterior, la población estudiantil usuaria del MOOC tendrá un compromiso con el aprendizaje y mayor fidelidad con las tareas, con el curso y con los participantes.

Por su parte Csikszentmihalyi y Csikszentmihalyi (1998) y Csikszentmihalyi (2003) en sus estudios sobre la felicidad ha desarrollado una teoría que resalta y describe la experiencia de flujo o flow -muy asociada a los juegos y a la gamificación- como un placer espontáneo mientras se desarrolla una tarea; es decir, como estar totalmente absorto por la actividad que se realiza, donde cada acción, cada movimiento y pensamiento sigue inevitablemente al anterior, lo cual implica la utilización de las habilidades al máximo. En el flujo hay ocho componentes clave:

1. Que suponga un reto y requiera destreza

2. Una fusión entre acción y consciencia

3. Objetivos claros. 
4. Retroalimentación directa e inmediata

5. Concentración en la tarea que se realiza

6. Sensación de control

7. Pérdida de la auto-consciencia

8. Distorsión del sentido del tiempo

Rosler (2015a, 2015b) y Logatt (2018) afirman que en educación para que un recuerdo se consolide en la memoria se necesita estar asociado a una emoción para que esa emoción provoque la unión de neuronas (sinapsis) que pueden llegar a ser tan fuertes que los recuerdos consigan perdurar durante un gran tiempo e incluso toda la vida. De acuerdo con lo anterior, se necesitan metodologías donde el alumnado se divierta, en las que sienta lo que aprende y lo conjugue en la creación del conocimiento (Guillén, 2017). Rosler (2015a), menciona que a través de la neurodidáctica se puede lograr emocionar y conectar los contenidos con el día a día del alumnado, para lo cual propone siete pasos para consolidar el aprendizaje: 1. Generar emoción, abrir las memorias sensoriales, para lo cual se pueden utilizar diversos elementos para hacer relevante la información o contenidos que se transmite. Es importante sorprender, despertar la curiosidad a través de historias datos, objetos y preguntas que generen desconcierto, emplear las metáforas, dibujos, imágenes, videos, música; 2. Generar reflexión, tras las dinámicas, la exposición de información o contenidos hay que generar un espacio y un tiempo para que las personas puedan pensar acerca de dichos contenidos, información o experiencia vivida; 3. Proponer un reto, 4. Propiciar la participación, 5. Impactar de corto a largo plazo, poner en acción todo lo aprendido de tal forma que permita hacer el tránsito de la memoria de trabajo a la memoria a largo plazo 6. La retroalimentación, realizar una evaluación de lo que se ha aprendido volver a trabajar sobre los contenidos en una forma diferente y 7. Recuperar, el sentido del aprendizaje es decir, hacer el conocimiento propio -las personas para aprender necesitan hacer suya la información recibida- (Rosler, 2015a, 2015b).

Para Sánchez, González y Prendes (2017), la potencialidad en la enseñanza de los juegos viene determinada por el reconocimiento de que su misión va más allá de puro entretenimiento, hay que tener en cuenta los objetivos, las reglas y los retos determinando el orden, los derechos y las responsabilidades del alumnado. Además, las personas deben enfrentarse con problemas a los que deben buscar solución. 
De acuerdo con lo anterior, la gamificación busca lograr un cambio en la actitud del alumnado, utilizando para ello elementos de juego que llamen su atención. Así que, antes de comenzar con la elaboración de un curso, es necesario concretar qué comportamientos se desea motivar, crear un contexto apropiado a esos fines y encontrar el sistema de recompensas que reconocerá el valor de las acciones que deseamos.

Chou (2015) desarrolló un marco conceptual de análisis estructurado llamado octalysis para lograr una mejor comprensión de la gamificación, señala ocho factores para motivar la implicación del usuario en un determinado contexto:

1. Sentido de llamada y significado épico: Este elemento de motivación se logra cuando el participante cree que ha sido elegido para jugar o que lo hace para lograr un fin loable.

2. Desarrollo y logro del deseo de progresar: Este elemento se refiere a la disposición natural a desear desarrollar competencias y superar desafíos.

3. Potenciación de la creatividad y retroinformación sobre sus resultados.

4. Propiedad y posesión: Impulsor que se da cuando el usuario siente que es propietario de algo.

5. Influencia social y afinidad: Incluye volverse mentor, la aceptación social, la respuesta del grupo y compañía, así como situaciones más negativas por ejemplo la envidia y la competencia.

6. Escasez e impaciencia: Este factor se enfoca en buscar algo que no se puede tener en el momento.

7. Curiosidad e imprevisibilidad: Impulsor que trata de averiguar lo que viene después, deseo de saber lo que ocurrirá.

8. Pérdida y evitación: Evitar que algo malo suceda, en especial cuando se trata de un esfuerzo acumulado que puede perderse al dejar de seguir realizando una acción.

En los cursos MOOC se diseñan espacios dirigidos a promover y estimular la comunicación entre las personas participantes y la creación de auténticas comunidades virtuales. En ese sentido, y para facilitar esa tarea, se diseñan actividades que estén muy vinculadas a problemáticas de la vida real, algo que facilita el intercambio y la discusión entre la comunidad (Gillani, 2013). La práctica de la educación creativa a través del uso de juegos digitales atrae a la población estudiantil, ya que contiene metas, reglas, conflictos, desafíos, retroalimentación y adaptabilidad que permite la inmersión, participación y la alta 
probabilidad de regresar. De acuerdo con lo anterior un MOOC debe favorecer un enfoque centrado en el estudiantado donde la participación-interacción y la motivación deben estar presentes durante su desarrollo (ProActive, 2018).

\section{Metodología}

\subsection{Enfoque de la Investigación}

La presente investigación tiene un enfoque mixto: cuantitativa y cualitativa, y un alcance descriptivo.

\subsection{Población de Estudio. Caracterización y contexto de la población}

En la investigación participaron 1906 estudiantes a nivel internacional, 31 expertos y 15 becarios que diseñaron y gestionaron la plataforma, así como 11 emprendedores que dieron su testimonio y colaboraron en la elaboración de algunos apartados del curso.

\subsection{Técnicas de recolección de la información}

La recolección de datos de todos los participantes se realizó a través de dos cuestionarios aplicados mediante la misma plataforma del curso.

\subsection{Procesamiento de análisis}

Se recabaron las respuestas de los cuestionarios y se analizaron las diversas variables, se utiliza estadística descriptiva. También se lleva a cabo la descripción de cómo se fue realizando el curso desde la planeación de cada uno de los módulos hasta la terminación del curso por parte del alumnado, así como el comportamiento de estos durante el curso.

\section{Resultados y discusión}

La idea del proyecto del curso MOOC de emprendimiento se desarrolla a través un Centro de Innovación Educativa (CIE) de la Universidad. Antes de iniciar el proyecto surgieron diversos cuestionamientos que influyeron en el impacto de cómo se iba a investigar y cómo se iba a concretar el proyecto.

Para el CIE, diseñar e implementar el MOOC resultó un gran reto y requirió de un proceso integral de investigación creativa y del involucramiento de 57 actores. Los actores que participaron en el proyecto son: 
- Veintitrés personas colaboraron en el diseño y gestión del curso

- Un Profesor Investigador, que fungió como coordinador del curso

- Seis Colaboradores (as) del Centro de Innovación Educativa (CIE) (facilitadores)

- Una Profesora del Centro de Lenguas

- Cinco becarios(as) del CIE

- Diez becarios(as) de la Escuela de Empreariales

- Once empresarios(as) y expertos(as)

El proyecto inicio con algunas preguntas: ¿Sobre qué tema se iba a tratar? ¿Qué plataformas ofrecen MOOC en el mundo? ¿Qué está sucediendo con los MOOC actualmente? ¿Qué se necesita para diseñar un MOOC? ¿Cuánto tiempo requiere? ¿En qué idioma? ¿Cómo hacerlo atractivo para que las y los estudiantes no lo abandonen? entre otras.

Finalmente se optó por un curso de emprendimiento en inglés, al cual le asignaron por nombre: Thinking Outside The Box: Creative Entrepreneurship, y cuyo objetivo es potenciar las habilidades de aquellas personas que tienen el deseo de emprender para idear y diseñar proyectos innovadores a través del pensamiento creativo. Se buscó que fuera una experiencia de aprendizaje compartida, es decir, una experiencia en la que se generara una red de conocimiento entre los participantes y donde destacaran las características siguientes:

- Fluidez y flexibilidad: abandonar fácilmente viejas formas de pensar para adoptar otras nuevas.

- Originalidad: llegar a ideas que están fuera de la lógica común.

- Asociaciones Remotas: formar asociaciones entre los elementos que, a primera vista, no parecen estar vinculados entre sí.

- Redefiniciones: utilizar objetos familiares de forma distinta.

- Sensibilidad ante los problemas: percibir las dificultades o deficiencias en instituciones bio-físicas, sociales o ambientales.

- Aceptación de ambigüedad: aceptar cierto grado de incertidumbre en las conclusiones.

- Divergencia: participar en el pensamiento indefinido, en donde no hay una sola respuesta correcta (Anders, Elvidge y Walsh, 2009).

- Aprendizaje: utilizar escenarios de aprendizaje basado en el juego es decir, la gamificación. 
El desarrollo del MOOC implicó una inversión en tiempo de diecinueve meses; catorce de ellos para la investigación, planeación y desarrollo del curso; dos meses para su ejecución; y tres meses para la documentación y análisis de resultados. En total, el curso se integró por siete etapas (ver Tabla 2).

Tabla 2.

Etapas de desarrollo para el curso MOOC: Thinking Outside The Box: Creative Entrepreneurship

\begin{tabular}{|c|c|}
\hline Etapa & $\begin{array}{r}\text { Actividades clave } \\
\end{array}$ \\
\hline $\begin{array}{l}1^{\circ} \text { Etapa } \\
\text { investigación previa }\end{array}$ & $\begin{array}{ll}- & \text { Investigación sobre los MOOC. } \\
\text { - } & \text { Investigación sobre el perfil de los participantes. } \\
\text { - } & \text { Investigación sobre las plataformas. }\end{array}$ \\
\hline $2^{\circ}$ Etapa desarrollo I & $\begin{array}{l}\text { - } \quad \text { Contrato de colaboración con Canvas Network. } \\
\text { - } \quad \text { Estructuración y planeación del curso. } \\
\text { - } \quad \text { Revisión de la propuesta del curso con diseñadora instruccional de } \\
\text { Canvas Network. } \\
\text { - } \quad \text { Desarrollo de identidad gráfica. }\end{array}$ \\
\hline $3^{\circ}$ Etapa desarrollo II & $\begin{array}{l}\text { - Desarrollo de los contenidos y estrategias de aprendizaje (que incluyó } \\
\text { planeación detallada de la gamificación). } \\
\text { - Revisión del idioma inglés. } \\
\text { - Revisión del primer módulo en la plataforma y retroalimentación por parte } \\
\text { la diseñadora instruccional de Canvas Network. }\end{array}$ \\
\hline $\begin{array}{l}4^{\circ} \text { Etapa diseño } \\
\text { gráfico y producción } \\
\text { multimedia }\end{array}$ & $\begin{array}{l}\text { - Diseño de pdf's interactivos para las lecturas del tema y actividades de } \\
\text { práctica guiada. } \\
\text { - Entrevistas a emprendedores y edición de videos. }\end{array}$ \\
\hline $\begin{array}{l}5^{\circ} \text { Etapa promoción } \\
\text { y publicación del } \\
\text { curso }\end{array}$ & $\begin{array}{l}\text { - Grabación y edición de un video de introducción, publicado en la página } \\
\text { inicial del curso en Canvas Network. } \\
\text { - Posters y postales de invitación publicados en la página web de la UP y } \\
\text { por correo institucional. } \\
\text { - Publicación en revistas y entrevista en el programa de radio. } \\
\text { - Revisión final y retroalimentación por parte de diseñadora instruccional de } \\
\text { Canvas Network. }\end{array}$ \\
\hline $6^{\circ}$ Etapa gestión & $\begin{array}{l}\text { - Reclutamiento, selección y capacitación de becarios de la Escuela de } \\
\text { Empresariales para apoyar en la gestión del curso como instructores } \\
\text { asistentes. } \\
\text { - Publicación de correos semanales de bienvenida, recordatorios, anuncios } \\
\text { y agradecimientos. } \\
\text { - Respuesta a comentarios y dudas de los participantes en los foros de } \\
\text { discusión en un plazo máximo de } 48 \text { horas. } \\
\text { - Acompañamiento a expertos durante su participación en los foros de } \\
\text { discusión y grabación de cápsulas de video respondiendo a las preguntas } \\
\text { más frecuentes e interesantes. } \\
\text { - Documentación de indicadores cuantitativos y cualitativos de cada } \\
\text { módulo, seguida de una junta semanal para revisar avances y realizar los } \\
\text { ajustes necesarios. } \\
\text { - Revisión de entrega de evidencias y envío de constancias. }\end{array}$ \\
\hline $\begin{array}{lr}7^{\circ} & \text { Etapa } \\
\text { documentación } & \text { y } \\
\text { análisis } & \text { de } \\
\text { resultados }\end{array}$ & $\begin{array}{l}\text { - Documentación y análisis de resultados finales y lecciones aprendidas } \\
\text { para la educación en línea. } \\
\text { - Producción de video con entrevistas a los involucrados en el proyecto } \\
\text { sobre su experiencia y aprendizajes. }\end{array}$ \\
\hline
\end{tabular}

Fuente: Elaboración propia con base en datos proporcionados por el CIE $(2015,2017)$. 


\subsection{Diseño y estructura del curso}

El diseño del curso se basó en una la metodología centrada en aprendizaje activo, al escalar las ideas de la población estudiantil a su al plan empresarial enfocado directamente en su producto / servicio y en el aprendizaje basado en juegos. El enfoque de aprendizaje activo permite desarrollar habilidades tales como: la mentalidad de diseño, la creación de sentido, el espíritu emprendedor, la detección y solución de problemas, la creatividad, el trabajo en equipo transdisciplinario y la innovación.

El MOOC analizado es de tipo cgMOOC, se basa en el conectivismo, en la cooperación y la gamificación, por lo que contó con diversos tipos de actividades en las que destacan:

1. Acceso de recursos atractivos y creativos.

2. Foros de discusión, redes sociales y comunidades de práctica.

3. Gamificación - diversos niveles de juego para avanzar.

4. Participación de diversos actores expertos en el tema, en la metodología pedagógica y en el idioma inglés.

5. Cooperación e interacción de todos los actores.

6. Una creación de autoría estudiantil que debía de compartirse con los demás.

El CIE utilizó estas características transversalmente durante el proceso de investigación que se generó para idear, diseñar e implementar el MOOC. El curso se creó en la plataforma tecnológica Canvas.net.

El objetivo del curso es analizar por parte de cada participante su idea de producto o servicio para diferenciarlo, al analizar su mercado potencial, al desarrollar estrategias de mercadotecnia, así como un primer borrador del plan de trabajo, para lo cual se utiliza lean canvas. El objetivo es llevar a la población estudiantil la idea de emprendimiento al diseño del producto y viabilidad de la creación, al hacer énfasis en que un buen producto debe satisfacer las demandas y las necesidades reales del cliente objetivo, considerando las características del mercado objetivo y con una propuesta de valor que integre intangibles, como calidad, estatus y prestigio, variedad de elección, servicio de seguimiento, personalización, etc.

El curso tiene cinco módulos temáticos para realizarse en ocho semanas. Para la realización del curso se emplearon métodos como la gamificación para la enseñanza en 
línea, con un enfoque en el aprendizaje, basado en actividades de juego y proyectos de aprendizaje. Cada módulo consiste en un nivel de juego. En cada nivel se encuentran las asignaciones que dan a conocer nuevos conceptos y desafíos que hay que cumplir para poder pasar al siguiente nivel. Además, se realizan debates interactivos con otras y otros estudiantes en foros, y se proporcionan infografías, artículos y vídeos que estimulan su reflexión, con el fin de percibir los cambios que ocurren en su aprendizaje.

El diseño y la estructura del curso se enfocaron en crear instrucciones precisas en los materiales, así como en todos los espacios de interacción, sobre lo que se esperaba de los participantes, así como sobre la forma de descargar o ingresar al contenido. En cuanto al diseño del juego -de gamificación-, se creó con la temática de emprendimiento y para el tipo de participante al que iba destinado -el emprendedor-. En el diseño de los materiales se consideró que su contenido fuera visualmente atractivo, que incluyera vídeos, imágenes, diagramas e infografías que le facilitaran al participante la comprensión de la estructura y lógica del contenido. También se elaboraron estudios de caso y actividades prácticas, las cuales tenían por objetivo propiciar un aprendizaje activo, así como estrategias para la aplicación del contenido y ejemplos de casos y experiencias que favorecerían el aprendizaje de la población estudiantil.

En cuanto a recursos adicionales, se realizó la inclusión de un compendio de recursos web en cada tema del curso, que permitiera profundizar en el tema de acuerdo con las inquietudes personales de los participantes. La producción de contenidos se presentó a través de cinco módulos de estudio. Se buscó que los participantes se identificaran con ser un emprendedor y con el proceso que vive desde el momento de tomar la decisión para convertirse en uno hasta la concreción de su empresa, contemplando el desarrollo de las competencias personales que se requieren para recorrer el camino. Los primeros módulos contemplan el análisis de habilidades que caracterizan a los emprendedores y las empresas más innovadoras del mundo. A partir del tercer módulo, lo aprendido se lleva a la práctica a través de la aplicación del proceso de creación del proyecto y de las herramientas para la detección de necesidades del mercado. Igualmente, en esta sección se analiza el desarrollo de un modelo de negocio creativo y las estrategias de venta innovadoras, como son el storytelling y el pitching. Como se puede observar en la tabla 3, cinco de los seis módulos contienen juegos -gamificación-. 
Tabla 3.

Mapa de aprendizaje: Módulos del curso de emprendimiento en MOOC

\begin{tabular}{|c|c|c|}
\hline $\begin{array}{l}\text { Actores que } \\
\text { participaron (57) }\end{array}$ & $\begin{array}{l}\text { Coordinador del cursc } \\
\text { creatividad, experto } \\
\text { Informáticos, diseñado }\end{array}$ & $\begin{array}{l}\text { pedagogos, traductores del Centro de lenguas, experto en } \\
\text { en marketing, empresarios, docentes, becarios, técnicos } \\
\text { es. }\end{array}$ \\
\hline Módulo & $\begin{array}{l}\text { Objetivo } \\
\text { módulo }\end{array}$ & Actividades y material del módulo \\
\hline $\begin{array}{r}\text { l. The } \\
\text { Journey of } \\
\text { Entrepreneurship }\end{array}$ & $\begin{array}{l}\text { Tener un panorama } \\
\text { del proceso } \\
\text { emprendedor y } \\
\text { comprender las } \\
\text { habilidades } \\
\text { personales } \\
\text { necesarias para } \\
\text { convertirse en un } \\
\text { empresario. }\end{array}$ & $\begin{array}{l}\text { 1. Leer: The Entrepreneur Roller Coaster } \\
\text { 2. Participar en el foro de discusión: The Thinking Corner } \\
\text { 3. Ver el video: Real world entrepreneurs: what are their } \\
\text { stories? } \\
\text { 4. Completar el ejercicio: Discover more stories y } \\
\text { compartir los hallazgos en el foro de discusión } \\
\text { 5. Completar: My Entrepreneurial Journal } \\
\text { 6. Compartir su: Entrepreneur } x \text {-ray y responder las } \\
\text { preguntas del foro de discusión: My entrepreneur } x \text {-ray }\end{array}$ \\
\hline $\begin{array}{r}\text { Meet the } \\
\text { creative } \\
\text { Entrepreneurs }\end{array}$ & $\begin{array}{l}\text { Entender el rol de la } \\
\text { creatividad para } \\
\text { tener éxito como } \\
\text { emprendedor. }\end{array}$ & $\begin{array}{l}\text { 1. Leer: The Entrepreneur's Creative Toolbox } \\
\text { 2. Participar en el foro de discusión con un experto invitado: } \\
\text { Any questions? } \\
\text { 3. Resolver el ejercicio: Warming up your creative muscles. } \\
\text { 1. Subir su PDF al foro de discusión. } \\
\text { 2. Ver el video: The thinking corner y participar en el foro } \\
\text { con otros participantes del curso. } \\
\text { 3. Completar: Let's play the memory game } \\
\text { 4. Compartir sus resultados de: Mix and Match en el foro de } \\
\text { discusión: }\end{array}$ \\
\hline $\begin{array}{l}\text { Detective } \\
\text { of needs }\end{array}$ & $\begin{array}{l}\text { Entender cómo se } \\
\text { identifican las } \\
\text { necesidades del } \\
\text { mercado dando } \\
\text { soluciones creativas. }\end{array}$ & $\begin{array}{l}\text { 1. Leer el documento: Entrepreneur: Detective of needs, } \\
\text { artist of solutions } \\
\text { 2. Ver la entrevista: The thinking corner y participar en el } \\
\text { foro de discusión. } \\
\text { 3. Completar la actividad: Market archaeologist: dig into } \\
\text { the real world } \\
\text { 4. Participar en el foro con un experto resolviendo dudas } \\
\text { respecto al plan de mercadeo. }\end{array}$ \\
\hline $\begin{array}{r}\text { IV. Sketching } \\
\text { your } \\
\text { Entrepreneurial } \\
\text { project }\end{array}$ & $\begin{array}{l}\text { Entender los } \\
\text { elementos creativos } \\
\text { de las empresas y } \\
\text { esbozar el primer } \\
\text { borrador de su plan } \\
\text { creativo de negocio. }\end{array}$ & $\begin{array}{l}\text { 1. Leer: The Innovative Company DNA } \\
\text { 2. Analizar algunas de las compañías más innovativas a } \\
\text { nivel mundial, ver la historia de los emprendedores e } \\
\text { identificar el ADN de su innovación. } \\
\text { 3. Ver y analizar algunas entrevistas a emprendedores, } \\
\text { identificando en el foro su ADN innovador, su historia de } \\
\text { éxito y algunas entrevistas con empresarios del mundo } \\
\text { real } \\
\text { 4. Trabajar en el foro: The thinking corner } \\
\text { 5. Leer: How to sketch your entrepreneurial project } \\
\text { 6. Completar la actividad: Back to our archaeological } \\
\text { findings } \\
\text { 7. Participar en el foro: My creative business model canvas } \\
\text { 8. Sesión de preguntas y respuestas que podrá aclarar un } \\
\text { experto, acerca de los tópicos relacionados con el } \\
\text { modelo de plan de negocio. }\end{array}$ \\
\hline
\end{tabular}




\begin{tabular}{|c|c|c|}
\hline $\begin{array}{r}\text { V. The next } \\
\text { step: selling your } \\
\text { idea }\end{array}$ & $\begin{array}{l}\text { Analizar las ideas y } \\
\text { elementos atractivos } \\
\text { del negocio, hacer } \\
\text { ideas y mensajes en } \\
\text { etiquetar Practicar } \\
\text { las } \quad \text { técnicas } \\
\text { creativas } \\
\text { marketing. }\end{array}$ & $\begin{array}{l}\text { 1. Leer: The next step: selling your idea y completar cada } \\
\text { cambio presentado. } \\
\text { 2. Votar en el foro por la mejor: Storytelling for creative } \\
\text { entrepreneurs } \\
\text { 3. Observar el video presentado en el foro: The thinking } \\
\text { corner y contestar las preguntas } \\
\text { 4. Investigar: hacer investigación de instituciones y } \\
\text { programas que apoyan el emprendimiento en tu } \\
\text { comunidad y participar en el foro: Entrepreneurship } \\
\text { around the world }\end{array}$ \\
\hline
\end{tabular}

Fuente: Elaboración propia con base en información proporcionada por el CIE-UP (2015).

Al inicio y al término del curso se les pidió a los participantes que llenaran unos cuestionarios, los cuales se procesaron con la finalidad de conocer el perfil de los participantes, sus expectativas, las inquietudes y lo que más les gusto. El análisis de los datos muestra lo siguiente: en el curso se registraron 1910 personas, de las cuales 890 (46.60\%) participaron al inicio y finalizaron 216 (24.3\%), y 11.30\% de los registrados. La eficiencia terminal fue alta (considerando lo mencionado en la revisión de la literatura $4 \%$ ). Los participantes se conectaron en diferentes husos horarios en más de 88 países de todo el mundo, aunque la mayoría de los participantes radican principalmente en América Latina, México, Estados Unidos, India, Canadá, Inglaterra y Ucrania. El 44\% de los participantes pertenecen a un rango de edad entre 21 y 30 años. Se les pidió que dedicaran de tres a cinco horas por semana para leer los contenidos, participar en los foros y realizar las actividades programadas (ver Tabla 4).

Tabla 4.

Perfil del alumnado en curso de Emprendimiento en MOOC

\begin{tabular}{|c|c|c|c|c|}
\hline Nacionalidad & Edad & Género & Nivel Educativo & $\begin{array}{l}\text { Motivo de } \\
\text { inscripción }\end{array}$ \\
\hline $\begin{array}{l}\text { - } 220 \text { estudiantes de } \\
\text { México } \\
\text { - } 86 \text { estudiantes de } \\
\text { Estados Unidos de } \\
\text { América } \\
\text { - } 29 \text { estudiantes de } \\
\text { Canadá } \\
\text { - } 21 \text { estudiantes de } \\
\text { India } \\
\text { - } 20 \text { estudiantes de } \\
\text { Ucrania } \\
12 \text { estudiantes } \\
\text { Inglaterra } \\
\text { - } 502 \text { estudiantes s } \\
\text { de otros países }\end{array}$ & $\begin{array}{l}\text { El rango de edad } \\
\text { es el siguiente: } \\
\text { De } 20 \text { años o } \\
\text { menos: } 13 \% \\
\text { De } 21 \text { a } 30 \text { años: } \\
44 \% \\
\text { De } 31 \text { a } 40 \text { años: } \\
19 \% \\
\text { De } 41 \text { a } 50 \\
\text { años: } 11 \% \\
\text { Más de } 50 \text { años: } \\
\text { 13\% }\end{array}$ & $\begin{array}{l}\text { Del total de los } \\
\text { estudiantes el } \\
47 \% \text { son de } \\
\text { género Masculino } \\
\text { y el } 53 \% \text { de } \\
\text { género Femenino }\end{array}$ & $\begin{array}{l}\text { - Preparatoria } \\
\text { - Estudios } \\
\text { inconclusos } \\
\text { - Licenciatura }\end{array}$ & $\begin{array}{l}\text { Los principales } \\
\text { motivos para } \\
\text { inscribirse en el } \\
\text { curso so: } \\
\text { I. Abrir su propio } \\
\text { negocio } \\
\text { II. Incrementar sus } \\
\text { conocimientos }\end{array}$ \\
\hline TOTAL 890 & $100 \%$ & $100 \%$ & & \\
\hline
\end{tabular}

Fuente: Elaboración propia con base en información proporcionada por el CIE-UP (2015) 
El total de personas conectadas durante las ocho semanas del curso fue de 890 . E total de horas de conexión durante las ocho semanas fue de 12673 , lo que equivale a un promedio de 14.23 horas por persona, que dista un poco del mínimo requerido de dedicación (de 3 a 5 horas por semana) por estudiante, de 18 a 30 horas en promedio. Cabe resaltar que hubo tres personas de sexo femenino que se conectaron más de 150 horas, teniendo un promedio de 31.06 horas por semana, cumpliendo las horas requeridas de dedicación.

El perfil del alumnado muestra que la mayor parte de estos fueron de México (220) seguido de Estados Unidos de América (86) y Canadá (29) (ver Tabla 4).

Dentro del cuestionario final aplicado, el resultado evidenció que lo que más valora el estudiantado de un MOOC son los materiales proporcionados (82.24\%), la interacción con expertos en los foros de discusión (54.39\%) y la interacción con otros participantes (35.26\%). El $48.56 \%$ de los participantes quieren abrir su propio negocio y el $25.15 \%$ desean mejorar en su trabajo actual. De acuerdo con estos datos obtenidos, se corrobora que al inicio deserta más de la mitad de las personas inscritas (53.40\%) (Adamopoulos, 2013) y permanecen solo 890 participantes. El porcentaje de la población activa, es decir, los que siempre estuvieron en todos los módulos y participaron en todas las actividades es de 24.3\%. Lo cual evidencia una tasa de retención arriba del mercado que, de acuerdo con MOOC-Maker (2017), es inferior al 10\% de deserción, sin embargo, continúa siendo baja.

Las fortalezas más relevantes del curso detectadas por los participantes son:

- Diseño y presentación creativa de la información

- Actividades de práctica guiada que incluyen los juegos de gamificación

- Calidad del contenido

- Recursos web adicionales en cada tema

- Interacción con expertos en los foros de discusión

- Videos de entrevistas con emprendedores

- Preguntas guía en los foros de discusión para formar una comunidad de diálogo

Las áreas de oportunidad del curso detectadas por los participantes son:

- Algunas actividades requerían de mucho tiempo para su realización

- Profundizar más en las entrevistas con los emprendedores

- Menor cantidad de actividades o más sencillas

- Dar más peso a las entrevistas con los emprendedores 
- Posibilidad de avanzar en el contenido y actividades de los módulos sin tener que esperar una semana.

De acuerdo con lo anterior, los elementos clave son: la gamificación, la interacción y la calidad del contenido. No obstante, se tiene que cuidar que el curso no contenga demasiada información y que el tiempo para impartirlo no se tan largo.

\section{Conclusiones}

Los formatos MOOC presentan enormes desafíos para los modelos de negocios de las instituciones de educación superior. Uno de estos desafíos para todos los involucrados en los MOOC, incluyendo a las grandes plataformas, es avanzar a un ritmo adecuado de cambio: seguir moviéndose lo suficientemente rápido para mantener el impulso, pero no tan rápido como para que muchos posibles participantes se incorporen.

La tendencia de los Cursos Masivos Abiertos en Línea (MOOC) están teniendo un fuerte impacto y presencia en la educación a distancia y han resultado particularmente importantes para la población estudiantil que necesita desarrollar un tipo de habilidades y/o aprender sobre temas relevantes como en este caso el curso de emprendimiento, más que para obtener algún grado académico y/o certificación.

La interacción social llevada acabo en los cursos MOOC a través de foros de debate y redes sociales ha sido muy positiva, y ofrece un espacio donde los participantes pueden expresarse libremente, exponer distintas ideas y puntos de vista de forma constructiva, dando lugar a la creación de comunidades de aprendizaje. Respecto a la gamificación es necesario hacer buen uso de ella, practicar, probar y experimentar cuál es la mejor forma de combinar los elementos utilizados para producir experiencias de juego motivadoras y llenas de contenido.

Cabe señalar que, de acuerdo con esta experiencia, se constata que la principal herramienta para estos cursos de emprendimiento no es el plan de negocios ni el financiamiento -aunque sí deben de conocer y tenerlo presente-, sino la creatividad empresarial. Por lo que, para una enseñanza efectiva del proceso emprendedor se tiene que partir de esta realidad y hacer un curso creativo que mueva el interés y la pasión por el emprendimiento del participante, donde resalte la utilización de la gamificación. Sin embargo, hay que considerar que la transferencia de conocimiento a través de ambientes virtuales es 
un proceso más elaborado que el presencial, que requiere de la participación de más actores y de más recursos como lo ejemplifica el caso presentado.

Para un aprendizaje más sostenido, se debe trabajar en aspectos de contenidos y estrategias MOOC; por ejemplo, en mecanismos de entrega de contenido, en la calidad del contenido, su presentación y en los tiempos.

Una limitación de la presente investigación para futuras investigaciones se enfoca en estudiar y analizar la efectividad pedagógica en relación con diferentes estructuras de diseño adaptadas a contenidos y a diferentes competencias que se desee alcanzar, y que permita dar respuesta a preguntas como: ¿qué es lo que aprenden los participantes en un MOOC?, ¿Cuál es el costo de cada desarrollo? ¿Cuál es el impacto en la marca institucional? ¿Se respetan las cuestiones éticas en los MOOC? Asimismo, se hace necesario poner en acción investigaciones que se enfoque en las nuevas teorias sugidas a partir de esta tecnología.

\section{Referencias}

Adamopoulos, Panagiotis. (2013). What makes a great MOOC? An interdisciplinary analysis of student retention in online courses. Thirty Fourth International Conference on Information Systems, Milan. Recuperado de http://pages.stern.nyu.edu/ padamopo/What\%20makes\%20a\%20great\%20MOOC.pdf

Álvarez, Guadalupe y Bassa, Lorena. (2013). TIC y aprendizaje colaborativo: el caso de un blog de aula para mejorar las habilidades de escritura de los estudiantes preuniversitarios. Revista de Universidad y Sociedad del Conocimiento, 10(2), 5-19. doi: http://dx.doi.org/10.7238/rusc.v10i2.1740

Anders, Katie, Elvidge, Liz y Walsh, Elaine. (2009). Doing creative research: A good practice guide for postgraduate researchers in STEM disciplines. London: Graduate School, Imperial College London. Recuperado de https://workspace.imperial.ac.uk/graduateschool/Public/Creative\%20Research/PG\%20i $\underline{\text { n\%20STEM.pdf }}$

Bayona, Cristina y González, Raquel. (2010). La transferencia de conocimiento en la universidad pública de Navarra. Una visión desde la empresa y desde el ámbito universitario (Proyecto SEJ2007-67737-C03-02 del Ministerio de Ciencia y Tecnología). Navarra, España: Ministerio de Industria, Turismo y Comercio y la Universidad Pública de Navarra.

Centro de Innovación Educativa de la Universidad Panamericana. (2015). MOOC "Thinking Outside the Box: Creative Entrepreneurship", CIE-UP. Recuperado: https://cie.up.edu.mx/profesores/proyectos/mooc-\%E2\%80\%9Cthinking-outside-boxcreative-entrepreneurship\%E2\%80\%9D 
Clark, Donald. (2013). MOOCs: taxonomy of 8 types of MOOC. Recuperado de http://donaldclarkplanb.blogspot.co.uk/search?q=MOOCs:+taxonomy

Csikszentmihalyi, Mihaly. (2003). Fluir. Una psicología de la felicidad (Nuria López, Trad.; 9a. ed.). Barcelona: Kairós.

Csikszentmihalyi, Mihaly y Csikszentmihalyi, Isabella. (1998). Experiencia óptima: Estudios psicológicos del Flujo en la Conciencia (J. Aldekoa, Trad.). Bilbao, España: Desclée de Brouwer.

Chou, Yu-Kai. (2015). Actionable Gamification: Beyond Points, Badges, and Leaderboards. Fremont, California: Octalysis Media.

Daniel, John. (2012). Making Sense of MOOCs: Musings in a Maze of Myth, Paradox and Possibility. Journal of Interactive Media in Education, (3). Doi: http://doi.org/10.5334/2012-18

Educase. (November 9, 2011). 7 Things you should know about MOOCs. Educase: learning initiative. Recuperado de https://library.educause.edu/resources/2011/11/7-things-youshould-know-about-moocs

Fidalgo Blanco, Ángel, Sein-Echaluce Lacleta, María Luisa, Borrás Gené, Oriol y García Peñalvo, Francisco José. (2014). Educación en abierto: Integración de un MOOC con una asignatura académica. Teoría de la Educación. Educación y Cultura en la Sociedad de la Información, 15(3), 233-255.

García Retana, José Ángel. (2011). Modelo educativo basado en competencias: importancia y necesidad. Revista Actualidades Investigativas en Educación, 11(3), 1-24. Doi 10.15517/aie.v11i3.10225.

García-Peñalvo, Francisco José, Fidalgo Blanco, Ángel, y Sein-Echaluce Lacleta, María Luisa. (2015). Tendencias en Innovación Educativa. Conferencia presentada en el III Congreso Internacional sobre Aprendizaje, Innovación y Competitividad (CINAIC 2015), Madrid, España. Recuperado de http://gredos.usal.es/jspui/handle/10366/126559

García-Peñalvo, Francisco José, Fidalgo-Blanco, Ángel y Sein-Echaluce, María Luisa. (2017). Los MOOC: un análisis desde una perspectiva de la innovación institucional universitaria. La Cuestión Universitaria, 9,117-135.

Gillani, Nabeel. (2013). Learner Communications in Massively Open Online Courses. Recuperado http://oxcheps.new.ox.ac.uk/MainSite\%20pages/Resources/OxCHEPS OP53.pdf

Guillén, Jesús. (2017). Una escuela con cerebro y corazón [Conferencia en Vigo organizada por Faro de Vigo]. En Canal Jesús C. Guillén [En línea]. Disponible en https://www.youtube.com/watch?v=dTTV7ZYvBjk 
Hernández-Carranza, Erika, Romero-Corella, Sandra y Ramírez-Montoya, María. (2015). Evaluación de competencias digitales didácticas en cursos masivos abiertos: Contribución al movimiento latinoamericano. Comunicar, 22(44), 81-90. https://doi.org/10.3916/C44-2015-09

Holi, Martin, Wickramasinghe, Rochana, y Leeuwen, Matthijs. (2008). Metrics for the evaluation of knowledge transfer activities at universities. United Kingdom: Library House. Recuperado de http://ec.europa.eu/invest-inresearch/pdf/download en/library house 2008 unico.pdf

Kapp, Karl. (2012). The Gamification of Learning and Instruction: Game-Based Methods and Strategies for Training and Education. San Francisco: John Wiley \& Sons.

Logatt, Carlos. (2018). ¿Cómo influyen las emociones en el aprendizaje? Revista de Neurociencias y Neurosicoeducación, (83), 6-8. Recuperado de https://www.upla.cl/inclusion/wpcontent/uploads/2016/05/Descubriendo el cerebro y la mente n83.pdf

Mcauley, Alexander, Stewart, Bonnie, Siemens, George y Cormier, David. (2010). The MOOC model for digital practice. Canada: University of Prince Edward Island. Recuperado de http://davecormier.com/edblog/wp-content/uploads/MOOC Final.pdf

MOOC-Maker. (2016). Construction of Management Capacities of MOOCs in Higher Education. WDP1.6. Deserción y permanencia en entornos MOOC. Erasmus+ de la Unión Europea MOOC-Maker. Recuperado de http://www.mooc-maker.org/wpcontent/files/WPD1.6 ESPAOL.pdf

Organización para la Cooperación y el Desarrollo Económicos. (2008). El conocimiento libre y los recursos educativos abiertos. París: OCDE, Junta de Extremadura.

Oriol-Borras. Gené. (2015). Fundamentos de la gamificación. Madrid: Gabinete de TeleEducación de la Universidad Politécnica de Madrid. Recuperado de http://oa.upm.es/35517/1/fundamentos\%20de\%20la\%20gamificacion v1 1.pdf

Oriol-Borras, Gené, Núñez, Margarita y Blanco, Ángel. (2014). Gamification in MOOC: challenges, opportunities and proposals for advancing MOOC model. Proceedings of the Second International Conference on Technological Ecosystems for Enhancing Multiculturality (pp. 215-220). ACM. Salamanca, España.

Pernías, Pedro y Luján, Sergio. (2014). Los MOOC: orígenes, historia y tipos. Historia, definición, tipología y valoración de los Massive Open Online Courses (MOOC). Revista Comunicación y Pedagogía, (Especial), 269-270.

Porter, Sarah. (2015). To MOOC or Not to MOOC How Can Online Learning Help to Build the Future of Higher Education? United Kingdom: Chandos Publishing.

Pomerol, Jean-Charles, Epelboin, Yves y Thoury, Claire. (2015). MOOCs Design, Use and Business Models. New Jersey, United States: John Wiley \& Sons, Inc. 
ProActive. (2018). Fomentando la creatividad: creación de escenarios de aprendizaje basados en juegos. Una guía para profesores. Recuperado de http://www.ub.edu/euelearning/proactive/documents/handbook creative gbl es.pdf

Ramírez Montoya, María Soledad. (2008). Formación de investigadores educativos a través de redes virtuales. El caso de la Cátedra de Investigación de Innovación en Tecnología y Educación del Tecnológico de Monterrey. México. Recuperado de http://recursos.portaleducoas.org/sites/default/files/10851.pdf

Renninger, K. Ann, Cai, Ming, Lewis, Mark, Adams, Margot y Ernst, Katherine. (2011). Motivation and learning in an online, unmoderated, mathematics workshop for teachers. Educational Technology Research and Development, 59(2), 229-247. Doi: 10.1007/s11423-011-9195-4

Rivera, Nohemi. (2014). La transferencia del aprendizaje: Un reto para los MOOC. En Congreso Internacional de Organización Escolar (XIII CIOIE). Madrid, España.

Rosler Roberto. (2015a). Clases cerebralmente amigables para que sus alumnos recuerden lo que usted les enseña (tercera parte). Revista de Neurociencias y Neurosicoeducación, (80), 33-39. Recuperado de https://www.upla.cl/inclusion/wpcontent/uploads/2015/06/Descubriendo el cerebro y la mente n80.pdf

Rosler Roberto. (2015b). Sin Neurotransmisores felices no hay aprendizaje. Revista de Neurociencias y Neurosicoeducación, (80), 50-65. Recuperado de https://www.upla.cl/inclusion/wpcontent/uploads/2015/06/Descubriendo el cerebro y la mente n80.pdf

Ruíz, Carlos. (2016). El MOOC: ¿un modelo alternativo para la educación universitaria? Apertura. Revista de Innovación Educativa, 7(2), 86-100.

Sánchez Vera, María del Mar, González Calatayud, Víctor y Prendes Espinosa, María Paz. (2017). Los MOOC y la evaluación del alumnado: revisión sistemática (2012-2016). @ tic. revista d'innovació educativa, (18), 65-73.

Siemens, George. (2015). The Role of MOOC's in the future of Education. In Curtis J. Bonk, Mimi M. Lee, Thomas C. Reeves and Thomas H.Reynolds (eds.), MOOCs and Open Education Around the World (pp. xiii-xvii). New York: Routledge, Taylor \& Francis Group.

SCOPEO. (2013). Mooc: Estado de la situación actual, posibilidades, retos y futuro. (Scopeo Informe $\quad \mathrm{N}^{\circ} \quad$ 2). Recuperado de http://scopeo.usal.es/wpcontent/uploads/2013/06/scopeoi002.pdf

Terán-Bustamante, Antonia y Chavarría, Olarte Marcela. (Octubre, 2014). El talento humano y la transferencia de Conocimiento: hacia un modelo educativo Innovador en la formación docente basado en competencias. XIX Congreso Internacional de Administración, Contaduría e Informática. México. 
Torres-Díaz, Juan, Infante, Alfonso y Valdiviezo, Pedro. (2014). Los MOOC y la masificación personalizada. Profesorado: Revista de curriculum y actualización al profesorado, 18(1), 63-72.

Uden, Lorna, Sinclair, Jane, Tao, Yu-Hui and Liberona, Dario (Eds.). (september, 2014). Learning Technology for Education in Cloud-MOOC and Big Data. Third International Workshop (LTEC 2014) Santiago, Chile.

Vázquez-Cano, Esteban y López Menese, Eloy. (2014). Los MOOC y la educación superior: la expansión del conocimiento. Revista Profesorado. Currículum y Formación del Profesorado, 18(1), 3-12.

Vázquez, Esteban, López, Eloy y Sarasola, José Luis. (2013). La expansión del conocimiento abierto: Ios MOOC. Barcelona, España: Octaed.

Vest, Charles. (2006). Open Content and the Emerging Global Meta-University. Educause review, 41(3), 18-30.

Werbach, Kevin y Hunter Dan. (2015). The Gamification Toolkit: Dynamics, Mechanics, and Components for the Win. Philadelphia: Wharton Digital Press.

Yee, María. (2012). La educación a distancia como un bien público: una visión. En veinte visiones de la educación a distancia. En Manuel Moreno Castañeda (coord.), Veinte visiones de la educación a distancia (pp. 95-109). México: Universidad de Guadalajara.

Zheng, Ye, y Yang, Ruo-Yu. (2017). The Rise of MOOCs: The Literature Review of Research Progress and Hot Spots of MOOCs Education in Mainland China. Eurasia Journal of Mathematics, Science and Technology Education, 13(9), 6165-6174. Doi: https://doi.org/10.12973/eurasia.2017.01056a

Zichermann, Gabe y Cunningham, Christopher. (2011). Gamification by Design: Implementing Game Mechanics. Cambridge, MA: O’Reilly Media. 
Revista indizada en

sciplo

redalyc. latindex

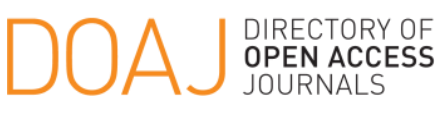

Distribuida en las bases de datos:

- Dialnet

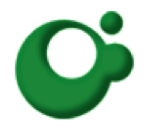
SHERPA/RøMEO

REDIB

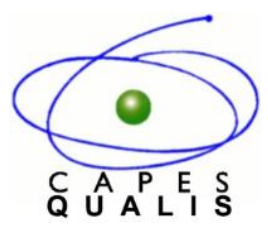

MIAR 